\title{
Efeitos da caminhada no sistema musculoesquelético - estudo da flexibilidade
}

\author{
Effects of walking in muscleskeletical system - \\ study of flexibility
}

\author{
Fátima Aparecida Caromano ${ }^{1}$, Rachel Rodrigues Kerbauy ${ }^{2}$, Clarice \\ Tanaka ${ }^{3}$, Maiza Ritomi Ide ${ }^{4}$, Cláudia Marchetti Vieira da Cruz
}

\begin{abstract}
CAROMANO, F. A.; KERBAUY, R. R.; TANAKA, C.; IDE, M. R.; CRUZ, C. M. V. Efeito da caminhada no sistema musculoesquelético - estudo da flexibilidade. Rev. Ter. Ocup. Univ. São Paulo,v. 18, n. 2, p. 95-101, maio/ago., 2007.
\end{abstract}

\begin{abstract}
RESUMO: Este estudo avaliou o efeito de um programa de exercício físico - caminhada - em pessoas idosas saudáveis, previamente sedentárias, na flexibilidade. Um programa de treinamento físico de baixa a moderada intensidade foi desenvolvido e testado. Foi estabelecido parâmetro clínico para classificar e avaliar a evolução da flexibilidade. O estudo foi realizado com 20 pessoas (10 em grupo experimental - A, e 10 em grupo de controle - B) com idade média 68,6 anos, ativos nas comunidades e livres de distúrbios ou doenças cardiovasculares, pulmonares, neurológicas ou musculares, ou ainda de doenças crônicas que poderiam afetar a habilidade para praticar exercícios. $\mathrm{O}$ treinamento ocorreu durante quatro meses, em sessões de uma hora, duas vezes por semana, alcançando $40 \%$ a $70 \%$ de freqüência cardíaca máxima calculada. Ao término do quarto mês, avaliou-se novamente a flexibilidade (pós-teste). O treinamento produziu melhora clínica e estatística para flexibilidade.
\end{abstract}

DESCRITORES: Atividade física. Envelhecimento. Exercício. Idoso. Sistema músculo-esquelético. Caminhada.

\footnotetext{
1 Profa Dra do Curso de Fisioterapia da Faculdade de Medicina da USP.

2 Profa Titular do Curso de Fisioterapia da Faculdade de Medicina da USP.

3 Profa Titular do Curso de Psicologia do Instituto de Psicologia da USP.

${ }^{4}$ Profa MS do Curso de Fisioterapia da Universidade Estadual do Norte do Paraná.

${ }_{5}$ Mestranda em Ciências da Reabilitação - FMUSP.

Endereço para correspondência: Curso de Fisioterapia da FMUSP. Laboratório de Fisioterapia e Comportamento - LaFi.Com. Rua Cipotânea, 51 - Cidade Universitária da USP. São Paulo - SP, CEP: 05360-000.
} 


\section{INTRODUÇÃO}

$\mathrm{O}$ aumento da proporção de idosos na população, constatado atualmente na maioria dos países, inclusive o Brasil, se por um lado revela melhoria na qualidade de vida, por outro gera, tanto na ordem social e econômica quanto na de saúde pública, sobrecargas que, por si só, justificam estudos preventivos de saúde que, ao mesmo tempo, visem o bem-estar desse segmento populacional. O treinamento de exercícios físicos durante o envelhecimento com o objetivo de amenizar as limitações orgânicas, é um tema contemporâneo estudado por diversas áreas de pesquisa.

O treinamento de exercícios físicos tem sido objeto de pesquisas relacionadas com a preservação ou recuperação parcial das funções orgânicas afetadas pelo envelhecimento; a prática de atividade física produz adaptações biológicas que propiciam a melhora no funcionamento de vários órgãos e sistemas e no desempenho de habilidades motoras, auxiliam na prevenção de várias doenças, normalizam o estado emocional e facilitam a socialização (BALADY et al., 1994).

O contrário, isto é, a manutenção de hábitos sedentários, independente da idade, propicia perdas na maioria dos sistemas orgânicos, compromete as habilidades motoras e deteriora a saúde (MILLS, 1994). Segundo Blair (1993), um número significativo de estudos apóiam a hipótese de que o sedentarismo se relaciona com aumento do risco de morbidade e mortalidade decorrentes do desenvolvimento de doenças crônicas.

Das perdas físicas, as mioarticulares são as mais fáceis de serem percebidas durante o envelhecimento, a exemplo do enfraquecimento muscular, cuja causa é multifatorial.

Além da diminuição da força muscular, outra perda mioarticular, percebida como a dificuldade em realizar movimentos amplos, está associada à diminuição da flexibilidade. Define-se flexibilidade como a amplitude do movimento voluntário de uma combinação de articulações num determinado sentido. A limitação da flexibilidade relaciona-se a perda de força muscular, presença de encurtamentos musculotendíneos, diminuição da elasticidade da pele e alterações morfológicas periarticulares, que predispõem ao aparecimento de doenças osteoarticulares, como artrites e artroses (PAYTON; POLAND, 1983). A coluna vertebral torna-se menos flexível em consequiência das alterações nos discos intervertebrais e deformidades nas vértebras. Os movimentos da coluna cervical e ombro são os mais atingidos pelo envelhecimento (BATTIE et al., 1987).
A perda de força e a diminuição da flexibilidade, associadas às alterações ósseas e/ou dos tecidos moles, promovem modificações no posicionamento dos segmentos corporais durante a sustentação do corpo, em bipedestação (postura) e no padrão de deambulação (marcha) (DANIELS; WORTHINGHAN, 1981).

As vantagens da prática de exercícios para idosos, ou seja, a relação entre as perdas orgânicas características do envelhecimento normal e os ganhos decorrentes da prática de atividade física, dependem de como se processa o envelhecimento e da rotina de exercício físico praticada. Sabe-se que os benefícios à saúde ocorrem mesmo quando a prática de atividade física é iniciada em uma fase tardia de vida, por pessoas sedentárias, sendo benéfica inclusive para portadores de doenças crônicas (MOREY et al., 1996).

Blair et al. (1992) realizaram um levantamento das informações publicadas nos últimos 30 anos sobre a relação entre o exercício físico e a saúde. Encontraram que a prática de atividade física de baixa a moderada intensidade (de intensidade entre $40 \%$ a $60 \%$ da capacidade máxima):

- correlacionava-se com prevenção ou melhora do quadro clínico de algumas das principais doenças associadas ao sedentarismo, como coronariopatias, diabetes, hipertensão arterial, hipercolesterolemia, acidente vascular cerebral, osteoporose, osteoartrite, câncer de próstata, mama e cólon intestinal;

- está associada à promoção de benefícios nutricionais, proporcionando a normalização do apetite, favorecendo a absorção de nutrientes e auxiliando na mobilidade intestinal;

- tem efeito positivo na resposta imunológica do organismo e pode melhorar a qualidade do sono.

A prática de exercícios físicos também reduz o risco da doença arterial coronária pela melhora da capacidade cardiopulmonar, da circulação miocárdica, do metabolismo cardíaco e do aprimoramento das propriedades mecânicas do coração. Age também sobre os fatores de risco para doenças como a hipertensão arterial, alteração dos níveis plasmáticos de glicose e insulina, obesidade e anormalidades no perfil lipoproteico (FALUDI et al., 1996).

De forma geral, a melhora da função musculoesquelética, decorrente da prática de exercícios físicos, consiste em normalização da relação entre tensão e comprimento dos músculos, no aumento de suprimento sangüíneo, na melhora do metabolismo muscular, no aumento na deposição de sais de cálcio ao longo das linhas de tração e compressão dos ossos envolvidos com a atividade física e no aumento na capacidade de os ligamentos e tendões se submeterem à força de tensão. Essas modificações melhoram a qualidade da contração 
muscular, a resistência óssea e a organização do equilíbrio, postura e marcha (THOMPSON, 1994).

Estuda-se a prescrição de exercícios físicos considerando-se sua modalidade, intensidade, frequiência, o tempo de duração da sessão e a situação de encaminhamento: desde a simples indicação à orientação cuidadosa para sua realização, treinamento, até o encaminhamento a um serviço especializado que acompanhe o idoso nessa tarefa.

Quanto à prática de uma atividade física de rotina, a indicação de corridas por trinta minutos, três vezes na semana, foi substituída a partir dos anos oitenta pela recomendação de andar, visando impedir lesões musculoesqueléticas ou sobrecarga inadequada do sistema cardiopulmonar. Essa atividade pode ser executada por qualquer pessoa, em qualquer idade. Para fins de orientação aceita-se que o passo deva ser rápido, e o aumento da distância percorrida progressivo, devendo atingir no máximo 6 mil metros, que podem ser percorridos em duas ou três etapas durante o dia (CAMARGO, 1982).

Os argumentos para motivar e manter o idoso em um programa de exercícios simples e pouco onerosos, a exemplo da caminhada, como visto, são muitos. No entanto, quando um profissional da saúde recomenda esta atividade, quais os valores, em medidas objetivas, ele pode utilizar para demonstrar claramente melhora na condição física, de tal maneira que possa utilizar estas melhoras como reforçadores na manutenção da prática de atividade física, sem ter que contar com equipamentos sofisticados? Para responder esta pergunta, organizou-se uma pesquisa para avaliar os efeitos da caminhada na função musculoesquelética. $O$ primeiro destes estudos apresentado aqui, avaliou a flexibilidade.

Com base no exposto acima, o objetivo deste estudo foi avaliar o efeito de um programa de caminhada na flexibilidade, utilizando equipamentos simples e rotinas de fácil replicação, em uma população de mulheres idosas.

\section{MÉTODO}

2.1 Participantes - Participaram do estudo 20 voluntários, 16 mulheres e 04 homens, com média de idade $68,7 \pm 3,5$ anos. Os participantes foram sorteados para integrar um dos dois grupos de estudo com 10 sujeitos cada: A (caminhada) ou B (controle).

Os idosos foram selecionados segundo os seguintes critérios: não fumante, socialmente ativo na comunidade, não ter praticado, nos últimos cinco anos, exercícios físicos ou caminhadas com percursos superiores a um quilômetro, mais de uma vez por semana; não apresentar disfunções de origem musculoesquelética, neuromotora ou cardiovascular, nem doença crônica impeditiva das atividades físicas a serem treinadas. Para participarem do estudo, considerou-se ainda sua disponibilidade de tempo, meios de transporte e aceitação da rotina de treinamento - que previa um mínimo de faltas, com justificativa e reposição da sessão - intenção de completar o treinamento, bem como a disponibilidade de colaborar no estudo, no caso de ser sorteado para o grupo controle, participando das avaliações, sem praticar exercícios físicos num prazo de quatro meses. Todos deram consentimento formal. Todos os participantes foram avaliados por exame clínico e funcional.

\section{Local}

O estudo foi realizado no Laboratório de Comportamento e Saúde do Instituto de Psicologia da USP e nos laboratórios de Fisioterapia do Centro de Docência e Pesquisa do Curso de Fisioterapia da Faculdade de Medicina da USP.

\section{Materiais e equipamentos}

Máquina fotográfica $\left(\right.$ Cannon $\left.{ }^{\circledR}\right)$ com tripé, balança com antropômetro (Filizzolla ${ }^{\circledR}$ ), paquímetro manual (Mitutoyo $\left.{ }^{\circledR}\right)$, régua, trena $(3 \mathrm{M} \circledast)$, transferidor, esquadro e adesivos.

\section{Procedimentos}

O programa de caminhada, com duração de 60 minutos, era realizada duas vezes por semana, em terreno plano e local coberto. O primeiro mês de treinamento foi desenvolvido nas seguintes etapas: os primeiros 15 minutos em passo normal (sem andar devagar, nem acelerado), associando exercícios respiratórios; nos 30 minutos subseqüentes, intercalavam-se 5 minutos de passo acelerado com 5 minutos de passo normal, até completar o tempo; nos últimos 15 minutos, semelhantes aos iniciais, a caminhada era realizada com passo normal. Nos demais três meses de treinamento, o grau de dificuldade foi aumentando e, no final, os participantes andavam 5 minutos normalmente no início e no final da atividade, caminhando rápido por 50 minutos - intercalando períodos de desaceleração do passo, caso sentissem necessidade.

A frequiência cardíaca dos participantes, durante as caminhadas, variou entre $40 \%$ e $70 \%$ da freqüência cardíaca máxima calculada, caracterizando exercícios de baixa a moderada intensidade. Antes e no final dos treinamentos, 
os participantes submeteram-se a avaliação de desempenho físico.

Para avaliar a flexibilidade, os participantes foram fotografados sobre uma base, portando pontos de referência anatômica, realizando o teste dedo-chão. Por meio das fotografias, mediu-se a distância punho-chão direita e esquerda e os ângulos de inclinação da pelve e de flexão dos joelhos, conforme estabelecido por Caromano et al. (1995). Segundo estes autores, o teste é realizado por estudo fotográfico padronizado dos indivíduos realizando o teste simples de distância dedo-chão (modificado para punho-chão), durante a flexão do tronco. Fornece a medida da distância punho-chão durante a flexão do tronco e informações sobre a conformação do tórax e mobilidade da coluna vertebral durante o teste. As distâncias são medidas diretamente nas fotografias, com um paquímetro e um esquadro, e o ângulo de deslocamento da pelve com esquadro e transferidor, através dos pontos de referência anatômica, marcados no corpo dos sujeitos com um adesivo branco de $1 \mathrm{~cm}$ de diâmetro. Os participantes se vestem com trajes de banho. Após a colocação dos adesivos para localização de referências anatômicas, os participantes são posicionados sobre a base de apoio (banco de ferro de $30 \mathrm{~cm}$ por $35 \mathrm{~cm}$, com $20 \mathrm{~cm}$ de altura, revestido com placas de borracha preta antiderrapante, demarcado com fitas adesivas brancas para colocação dos pés em posições padronizadas. Nas bordas do banco colase fita adesiva branca que serve como referência de chão e direção para os participantes levarem as mãos.

Fotografa-se cada participante nas vistas lateral direita e frontal, em flexão do tronco, com joelhos estendidos, não sendo permitida sua flexão ou hiperextensão nem deslocamento posterior de pelve. A cabeça posiciona-se relaxada e os dedos das mãos apontam em direção à linha branca colada na base de apoio.

A máquina fotográfica deve ser colocada sobre um tripé, posicionada a 3 metros de distância do sujeito, e a altura é regulada para cada sujeito de tal forma que o centro da foto coincida com o umbigo da pessoa fotografada.

Obtida a fotografia, procede-se ao estudo da flexão do tronco e da distância punho-chão direita e esquerda. Para obter a medida do ângulo de deslocamento da pelve durante o teste de flexibilidade, traçam-se duas retas, com o auxílio de régua e esquadro: uma reta $a$ vertical, perpendicular à linha branca do suporte, passando pelo ponto anatômico do maléolo lateral; e uma reta $b$, começando no ponto do maléolo lateral e passando pelo ponto do trocânter maior do fêmur.
O ângulo de deslocamento da pelve é lido com o uso de um transferidor, posicionando seu eixo 0-180 graus sobre a linha branca do suporte com centro no ponto 1 , medindo-se o ângulo 'alfa' formado pelas retas a e b. O posicionamento normal da pelve durante a flexão é de $90^{\circ}$ com a horizontal. As alterações são medidas a partir de $90^{\circ}$ : o sujeito que apresenta ângulo maior que este, como por exemplo $96^{\circ}$, desloca a pelve posteriormente em $6^{\circ}$; aquele que apresenta $84^{\circ}$, desloca a pelve anteriormente em $6^{\circ}$. Deslocamentos superiores a cinco graus invalidam a realização da medida punho-chão, pois indicam a presença de compensação. Na fotografia em vista frontal mede-se o nivelamento dos membros inferiores.

Utiliza-se o paquímetro para medir a distância punhochão, entre o processo estilóide da ulna e a linha do suporte. Para calcular a distância real, a partir da foto, efetua-se uma regra de três, considerando duas medidas conhecidas, como a medida real e a fotografada da lateral do banco de suporte e a medida fotografada da distância punho-chão; o resultado da proporção será a medida real da distância punho-chão.

\section{Análise de dados}

A análise clínica foi realizada considerando-se cada participante individualmente, com base em critérios estabelecidos, que permitiu determinar melhora, piora ou manutenção do desempenho. Com base na análise de cada participante, descreveu-se o desempenho dos grupos.

A análise estatística foi realizada por meio do estudo de variância ANOVA 1-way, para determinar a significância das diferenças nos grupos, utilizando-se o teste de Kruskall-Wallis.

\section{RESULTADOS}

\section{Características dos participantes no pré-teste, por} grupos

A Tabela 1 apresenta os resultados referentes à idade, podendo-se constatar que a média de idade dos dois grupos eram significativamente semelhante $(\mathrm{p}=0,987)$. Os valores encontrados nos testes de avaliação do desempenho físico, submetidos ao Teste de Kruskal-Wallis ANOVA/ 1 Way, para verificar se existe diferença entre diferentes grupos num mesmo tempo, mostram que $\mathrm{A}$ iniciou o treinamento apresentando flexibilidade aumentada, isto é, menor distância punho-chão durante o teste, quando comparado aos grupos B. 
Tabela 1 - Média de idade dos participantes no pré-teste, por grupo.

\begin{tabular}{l|l|l}
\hline \multicolumn{3}{c}{ Idade (em anos) } \\
\hline Análise & $\begin{array}{l}\text { GrupoA } \\
\mathrm{n}=10\end{array}$ & $\begin{array}{l}\text { Grupo B } \\
\mathrm{n}=10\end{array}$ \\
\hline $\begin{array}{l}\text { 1. Média da idade e } \\
\text { desvio padrão }\end{array}$ & $68,4 \pm 3,7$ & $68,8 \pm 3,3$ \\
$\begin{array}{l}\text { 2. Diferença entre os 2 grupos } \\
\text { na mesma situação* }\end{array}$ & $\begin{array}{l}\text { Pré- teste: Não significante, } \\
\mathrm{p}=0,987\end{array}$ \\
\hline
\end{tabular}

* Pesquisada através do Kruskal-Wallis 1-Way-Anova Test

\section{Flexibilidade}

Considerando-se os critérios de evolução estabelecidos como sendo: melhora da flexibilidade - quando a distância dedo-chão diminuía em $10 \mathrm{~cm}$ ou mais, piora da flexibilidade - quando a distância aumentava em $10 \mathrm{~cm}$ ou mais e manutenção da flexibilidade - quando a variação da medida ficava entre 1 e $9 \mathrm{~cm}$.

Somente sete participantes do grupo A apresentaram melhora: a média da distância punho-chão do grupo diminuiu significativamente de $27,2 \mathrm{~cm}$ para $13,4 \mathrm{~cm}$ $(p=0,0051)$ (Tabela 2$)$.

Tabela 2 - Efeitos do treinamento no desempenho durante o teste de flexibilidade (comparação do pré-teste com o pós-teste).

\begin{tabular}{|c|c|c|c|c|}
\hline \multirow{3}{*}{$\begin{array}{l}\text { Medida } \\
\text { Distância punho-chão } \\
(\mathrm{cm})\end{array}$} & \multicolumn{4}{|c|}{ Teste de flexibilidade } \\
\hline & \multicolumn{2}{|c|}{ Análises } & \multicolumn{2}{|c|}{ Grupo A Caminhada } \\
\hline & Evolução & $\begin{array}{l}\text { Melhora } \\
\text { Piora } \\
\text { Manutenção }\end{array}$ & \multicolumn{2}{|c|}{10 de 10 participantes } \\
\hline \multirow{5}{*}{$\begin{array}{l}\text { Nivelamento de membros } \\
\text { superiores }(\mathrm{cm})\end{array}$} & \multicolumn{2}{|c|}{$\begin{array}{l}\text { Análise estatística } \\
\text { 1. média e desvio padrão }\end{array}$} & $\begin{array}{l}\text { Pré-teste } \\
39,2 \pm 13,1\end{array}$ & $\begin{array}{l}\text { Pós-teste } \\
36,9 \pm 11,9\end{array}$ \\
\hline & \multicolumn{2}{|c|}{ 2. diferença entre valores do pré-teste e do pós-teste } & \multicolumn{2}{|c|}{$\begin{array}{r}\text { Significativa } \\
\mathrm{p}=0,0051\end{array}$} \\
\hline & Evolução & $\begin{array}{l}\text { Melhora } \\
\text { Piora } \\
\text { Manutenção }\end{array}$ & \multicolumn{2}{|c|}{$\begin{array}{l}5 \text { de } 10 \text { participantes } \\
-- \\
5 \text { de } 10 \text { participantes }(4 \mathrm{com} \\
\text { nivelamento ótimo no PT) }\end{array}$} \\
\hline & \multicolumn{2}{|c|}{$\begin{array}{l}\text { Análise estatística } \\
\text { 1. média e desvio padrão }\end{array}$} & $\begin{array}{l}\text { Pré-teste } \\
0,9 \pm 1,0\end{array}$ & $\begin{array}{l}\text { Pós-teste } \\
0,3 \pm 0,5\end{array}$ \\
\hline & \multicolumn{2}{|c|}{ 2. diferença entre valores do pré- teste e pós-teste* } & \multicolumn{2}{|c|}{$\begin{array}{c}\text { Significativa } \\
\mathrm{p}=0,0277\end{array}$} \\
\hline
\end{tabular}

* Realizada através do Wilcoxon Matched-Pairs Signed-Ranks Test

Quanto ao nivelamento de membros superiores, para o estudo do nivelamento entre os membros superiores, utilizaram-se os seguintes critérios: nivelamento aceitável diferença entre os membros de até $1 \mathrm{~cm}$; nivelamento ótimo - diferença entre os membros de até $0,5 \mathrm{~cm}$ e desnivelamento - diferença entre os membros maior que $1 \mathrm{~cm}$.

Sete dos dez participantes avaliados apresentaram melhora com o treinamento de exercícios gerais, segundo os parâmetros de evolução. Dos outros três participantes, um apresentou piora e dois, que mantiveram o quadro, não poderiam apresentar melhora, pois partiram de um estágio de nivelamento ótimo. A diferença entre os dados encontrados no pré-teste e no pós-teste imediato foi significativamente diferente e as médias das distâncias entre o membro superior direito e esquerdo passaram de $1,28 \mathrm{~cm}$ para $0,35 \mathrm{~cm}$ (Tabela 2 ).

\section{DISCUSSÃO}

A utilização de fotografias para avaliação da flexibilidade revelou-se adequada, pois permitiu a observação e mensuração cuidadosa, sem requerer do participante esforço maior (por exemplo, permanência por período prolongado na posição de teste). 
Foi necessário estabelecer parâmetros de melhora, piora ou preservação das funções estudadas, para cada uma das medidas pesquisadas, pois essas informações, além de escassas, encontram-se incompletas na literatura. Para elaboração da tabela de evolução clínica, foram utilizadas informações de pesquisas publicadas nas áreas médica e de fisiologia do exercício físico e em experiências dos Laboratórios de Fisioterapia do Curso de Fisioterapia da USP. No intuito de validar essa tabela, foram pareados os resultados encontrados com os gerados pela análise estatística. Encontrou-se compatibilidade em $94 \%$ dos resultados obtidos, o que se considera satisfatório. A incompatibilidade foi resultado de um nível de exigência maior por parte dos critérios da tabela que os considerados na análise estatística realizada utilizando-se o teste Wilcoxon matched-pairs signed-ranks test. Este conjunto de instrumentos poderá auxiliar na rotina na avaliação física de idosos.

A melhora significativa, tanto clínica quanto estatística, encontradas neste estudo, são compatíveis com as informações produzidas por Rauchbach (1990). Esta autora treinou exercícios gerais associados à caminhada em 42 idosos com média de 64 anos, por três meses, três vezes por semana, mostrando que essas atividades produziram melhora da flexibilidade da coluna lombar e amplitude de movimentos das grandes articulações em 37 participantes $(88,2 \%)$. Nossos resultados mostraram também que a prática de caminhadas foi eficiente em produzir a normalização do nivelamento dos membros superiores durante o teste de flexibilidade. Associa-se à melhora do nivelamento com a melhora da função da musculatura posterior do tronco, dos membros inferiores e dos ombros, além de seu equilíbrio entre os hemicorpos direito e esquerdo. Não foram encontrados estudos similares na literatura, o que dificulta qualquer tipo de comparação.

No que diz respeito especificamente ao teste de flexibilidade, sugerimos coletar também a medida punhochão com o participante sentado, com os membros inferiores em extensão e pés em flexão dorsal, pois esse teste fornece informações complementares sobre possíveis encurtamentos da musculatura posterior dos membros inferiores e pelve; coletar, por meio de fotografia, a medida da envergadura, que fornece dados complementares sobre possíveis encurtamentos da cadeia ântero-interna dos membros superiores; avaliar em detalhe a mobilidade cervical, uma vez que esta afeta o equilíbrio, a marcha e a postura; explorar a inclinação lateral e a rotação do tronco, gerando dados para análise da mobilidade torácica.

\section{CONCLUSÃO}

No sistema músculo esquelético, o programa de caminhada treinado provocou melhora na flexibilidade, diminuindo tanto a distância punho-chão quanto o desnivelamento dos ombros.

CAROMANO, F. A.; KERBAUY, R. R.; TANAKA, C.; IDE, M. R.; CRUZ, C. M. V. Effect of walking in muscleskeletical sistem - study of flexibility. Rev. Ter. Ocup. Univ. São Paulo, v. 18, n. 2, p. 95-101, maio/ago., 2007.

\begin{abstract}
This study aimed at assessing the effect of program of physical exercise - walking - in healthy, previously sedentary old people, in flexibility. A program of low to moderate intensity physical training (walking) were developed and tested, As well a protocol to classify and assess the evolution of flexibility. The study was carried out with 20 people (10 in experimental group - A, and 10 in control group-B) aged in average 68,6 years, active in their communities, and free from significant cardiovascular, pulmonary, neurological or muscle disfunction, or from chronic diseases that might affect the ability to practice exercises. The training happened for four months, in twice-weekly sessions of one hour, reaching $40 \%$ to $70 \%$ of estimated maximum heart rate. At the end of the four-month training all participants had their flexibility evaluated (post-test). Training have brought flexibility clinical and statistical improvement.
\end{abstract}

KEY WORDS: Motor activity. Aging. Exercise. Aged. Musculoskeletal system. Walking.

\title{
REFERÊNCIAS
}

BALADY, G. J.; FLETCHER, C. B. J.; FROELICHER, E. S.; HARTLEY, L. H.; KRAUSS, R. M.; OBERMAN, A.;
BOLLOCK, M. L.; TAYLOR, B. Cardiac rehabilitation. Am. Heart Assoc. Circul., v. 90, n. 3, p. 1602-1610, 1994. 
BATTIE, M. C.; BIGOS, S. J.; SHEEHY, A.; WORTLEY, M. D. Spinal flexibility and individual factors that influence it. Phys. Ther., v. 67, n. 5, p. 653-658, 1987.

BLAIR, S. N.; KOHL, H. W.; GORDON, N. F.; PAFFENBARGER, R. S. J. R. How much physical activity is good for health? Annu. Rev. Publ. Health, v. 13, p. 99-126, 1992.

BLAIR, S. N. Physical activity, physical fitness and health. Res. Quar. Exerc. Sports, v. 64, n. 4, p. 365-376, 1993.

CAMARGO, R. B. A. Evite o enfarte praticando "Cooper". 7a ed. Rio de Janeiro: Parma, 1982.

CAROMANO, F. A.; OSTERMAYER, E.; TANIGUCHI, C.; NONAKA, L.; FARIAS, M. R. Flexibility and posture in elders using analyse photographic method. In: CONGRESSO PANAMERICANO DE GERONTOLOGIA,1., 1995, São Paulo.

DANIELS, M. A. L.; WORTHINGHAN, C. Provas de função muscular - técnicas de exame manual. 3a ed. São Paulo: Interamericana, 1981.

FALUDI, A. A.; MASTROCOLLA, L. E.; BERTOLAMI, M.
Atuação do exercício físico sobre os fatores de risco para doenças cardiovasculares. Rev. Soc. Cardiol. Est. São Paulo, v. 1, n. 6, p. $1-5,1996$.

MILLS, E. M. The effect of low intensity aerobic exercise on muscle strength, flexibility and balance among sedentary elderly persons. Nurs. Res., v. 43, n. 4, p. 207-211, 1994.

MOREY, M. C.; PIEPER, C. F.; SULLIVAN, R. J. J. R.; CROWLEY, G. M.; COWPER, P. A.; ROBBINS, M. S. Fiveyear performance trends for older exercise: a hierarchical model of endurance, strength, and flexibility. J. Am. Geriatr. Soc., v. 44. n. 10, p. 1226-1231, 1996.

PAYTON, O. D.; POLAND, J. L. Aging process: implications for clinical practice. Phys. Ther., v. 63, n. 1, p. 41-48, 1983.

RAUCHBACH, R. A atividade física para a terceira idade. Curitiba: Lovise, 1990.

THOMPSON, L. V. Effects of age and training on skeletal muscle physiology and performance. Phys. Ther., v. 74, n. 1, p. 71-81, 1994. 